Gastroenterologe 2022 $17: 71-73$

https://doi.org/10.1007/s11377-022-00595-x Angenommen: 12. Januar 2022

Online publiziert: 9 . Februar 2022

(C) The Author(s), under exclusive licence to Springer Medizin Verlag GmbH, ein Teil von Springer Nature 2022

\section{Innovative Endoskopie}

\author{
Arthur R. Schmidt ${ }^{1} \cdot$ Michael Fried $^{2} \cdot$ Ralf Jakobs $^{3}$ \\ ${ }^{1}$ Medizinische Klinik II, Universitätsklinikum Freiburg, Freiburg, Deutschland \\ ${ }^{2}$ International Office, Universitätsspital Zürich, Zürich, Schweiz \\ ${ }^{3}$ Medizinische Klinik C, Klinikum der Stadt Ludwigshafen, Ludwigshafen, Deutschland
}

Die Endoskopie hat sich im Laufe der letzten Jahre rasant weiter entwickelt. Neben verbesserten Technologien in der Bildgebung und der computerunterstützten Diagnostik mithilfe sog. künstlicher Intelligenz (KI) gibt es auch spannende Neuerungen im Bereich der Dünndarmendoskopie und der "drahtlosen“ Diagnostik. Technische und methodische Innovationen im Bereich der therapeutischen Endoskopie haben zudem das Indikationsspektrum für endoskopische Interventionen deutlich erweitert. Unter dem Titel „Innovative Endoskopie" stellen Experten den aktuellen Stand verschiedener endoskopischer Themenbereiche dar und geben an der einen oder anderen Stelle auch einen Ausblick in die Zukunft.

Thomas Brunk erläutert spannende Entwicklungen im Bereich der Kapseldiagnostik. Die Videokapselendoskopie ist seit 20 Jahren für die Dünndarmdiagnostik etabliert und wird überwiegend zur Diagnostik der mittleren gastrointestinalen (Gl-)Blutung eingesetzt. Auch außerhalb des Dünndarms scheinen weitere Einsatzgebiete in den Fokus der Videokapsel zu gelangen. Die neuesten Kapselgenerationen wurden hinsichtlich Auflösung, Blickwinkel und Batterienutzung deutlich verbessert und versprechen so eine höhere Untersuchungsqualität. Die computergestützte Befundung mit Einbindung von Kl-Applikationen und steuerbare Videokapseln dürften bald etabliert werden. Neben der "klassischen“ Videokapsel gibt es neuerdings auch ein Kapselsystem, das nach einem photometrischen Messprinzip Blut im oberen und mittleren Gl-Trakt detektieren und damit bei klinischen Therapieentscheidungen helfen kann. Diese Technologieplattform könnte in Zukunft als Basis für weitere, z. B. funktionelle Fragestellungen dienen.

》) Die flexible Enteroskopie ermöglicht neben der Diagnostik auch die Therapie von Dünndarmläsionen

Die flexible Enteroskopie ermöglicht neben der Diagnostik auch die Therapie von Dünndarmläsionen und kann zudem zur endoskopischen retrograden Cholangiopankreatikographie (ERCP) bei voroperierten Patienten eingesetzt werden. Björn Lewerenz fasst den aktuellen Stand zur "deviceassistierten Enteroskopie $^{\prime \prime}$ systematisch zusammen. Neben den klassischen Single- und Doppelballonsystemen hält zunehmend die motorisierte Spiralendoskopie Einzug. Erste Erfahrungen und frühe klinische Studien weisen bei geringerem Zeitaufwand auf hohe Eindringtiefen, hohe Raten an kompletten Resektionen und auch auf eine Erleichterung bei Interventionen durch die stabile Geräteposition hin. Bei aller Euphorie bleiben allerdings vergleichende Studien abzuwarten. Zudem sind Besonderheiten bei der Sedierung und auch Kontraindikationen zu beachten, die im Artikel praxisnah diskutiert werden.

Franziska Franck, Marie-Christine Becker und Markus Dollhopf stellen systematisch die Möglichkeiten und Grenzen von endosonographischen Interventionen dar. Auch hier haben sich in den letzten Jahren neue Indikationen ergeben. Endoskopisch-sonographische (EUS-)Rendezvousverfahren ermöglichen einen eleganten Zugang zum biliopankreatischen System bei nicht möglicher ERCP. Die EUSgesteuerten Anastomosen, wie die Hepatikogastrostomie, die Choledochoduode- 
nostomie sowie die Gastroenterostomie, sind insbesondere im palliativen Setting extrem attraktive Techniken, da dem Patienten externe Drainagen oder palliative Operationen erspart werden können. Sie können aber auch im Rahmen einer "Sequenztherapie" eingesetzt werden und so den Zugang zur Papille bei benignen Erkrankungen ermöglichen.

Subepitheliale Tumoren sind häufige Zufallsbefunde in der gastroenterologischen Praxis. Neben der Problematik der histologischen Sicherung steht der Arzt zunächst vor der Entscheidung, ob eine Surveillance oder eine Resektion indiziert ist. Benjamin Meier und Karel Caca nähern sich dieser Frage und diskutieren etablierte und neue endoskopische Resektionstechniken im Kontext aktueller Studienergebnisse.

Last, but not least stellen Tobias Blasberg und Edris Wedi innovative Instrumente und Techniken zur endoskopischen Resektion von Frühkarzinomen im Gl-Trakt vor. Hierbei konzentrieren sich die Autoren auf die endoskopische Submukosadissektion (ESD), die - wie aktuelle Ergebnisse des deutschen ESD-Registers zeigen - nun auch außerhalb der asiatischen Welt Einzug in die klinische Routine an größeren Zentren erhalten hat. Die Technik ist bekanntermaßen komplex und zeitaufwändig, kann aber durch technische Hilfsmittel erleichtert werden. Blasberg et al. stellen verschiedene Systeme dar, die einen "Gegenzug" bei der Resektion ermöglichen, und vergleichen verschiedene etablierte und neue Substanzen zur submukosalen Injektion. Für die praktische Anwendung und Komplikationsvermeidung werden auch Verfahren zur Prävention von sekundären Blutungen und Perforationen diskutiert.

Wir hoffen, Ihnen mit dieser Themenauswahl einen Einblick in aktuelle und künftige Innovationen der diagnostischen und interventionellen Endoskopie zu geben, und wünschen viel Freude beim Lesen.
Korrespondenzadresse

Prof. Dr. Arthur R. Schmidt

Medizinische Klinik II, Universitätsklinikum Freiburg

Hugstetter Str. 55, 79106 Freiburg, Deutschland arthur.schmidt@uniklinik-freiburg.de

Prof. Dr. Michael Fried

International Office, Universitätsspital Zürich Rämistr. 100, 8091 Zürich, Schweiz michael.fried@usz.ch

Prof. Dr. Ralf Jakobs

Medizinische Klinik C, Klinikum der Stadt Ludwigshafen

Bremserstr. 79, 67063 Ludwigshafen,

Deutschland

jakobsr@klilu.de

Interessenkonflikt. A. Schmidt: Vortragshonorare und Unterstützung klinischer Studien von Ovesco Endoscopy. R. Jakobs: Vortragshonorar von Boston Scientific, Projektunterstützung von Erbe Elektromedizin. M. Fried gibt an, dass kein Interessenkonflikt besteht.

\section{Hepato Update \\ Topaktuell und praxisnah}

6. und 7. Mai aus Berlin

Umfangreiches Update-Wissen so flexibel wie noch nie: Erstmalig findet das Hepatologie-Update-Seminar als Hybridveranstaltung statt. Bilden Sie sich vor Ort mit Kolleginnen und Kollegen oder bequem von zuhause aus fort die Zertifizierung ist für beide Formate beantragt.

Das Update-Konzept: Die wichtigsten Neuerungen des vergangenen Jahres aus der Hepatologie und hepatobiliären Chirurgie werden kritisch selektiert, analysiert und zusammengefasst. Die Relevanz für Ihren Klinik- und Praxisalltag steht dabei im Vordergrund und der ausführlichen Diskussion mit den Referierenden wird viel Raum gegeben.

In diesem Jahr werden neben den Kerngebieten der Hepatologie die Hot Topics »Leber im Rahmen von Systemerkrankungen « und »Lebertransplantation« vorgestellt.

Zum Gesamtpaket der Teilnahme gehören neben den umfangreichen Seminarunterlagen der Download aller Vortragspräsentationen und die Vorträge im Nachgang als Video-on-Demand auf der Videoplattform »streamed-up.com«.

Weitere Informationen zu Programm, Referierenden und zur Anmeldung: www.hepato-update.com

Veranstalter: med update $\mathrm{GmbH}$ Hagenauer Straße 53 65203 Wiesbaden 
Hier steht eine Anzeige.

黑 Springer 